\title{
УВЕЛИЧЕНИЕ ЭКСТРЕМАЛЬНЫХ ЗНАЧЕНИЙ ПРЕДЕЛА ПРОЧНОСТИ ОРГАНОПЛАСТИКОВ ПРИ ОБРАБОТКЕ АРАМИДНОГО ВОЛОКНА МНОГОСЛОЙНЫМИ УГЛЕРОДНЫМИ НАНОТРУБКАМИ
}

\author{
М.С. Шебанов ${ }^{1}$, В.Б. Иванов ${ }^{1}$, И.Г. Калинина ${ }^{1}$, В.Г. Бова ${ }^{2}$, Л.Б. Шиянова ${ }^{2}$ \\ ${ }^{1}$ Отдел динамики химических и биологических процессов. ФГБУН ФИЦ химической физики им. Н.Н. Семенова \\ РАН, ул. Косыгина, д.4, Москва, Российская Федерация, 119991 \\ E-mail: shebanov1989@gmail.com,vik93973@yandex.ru,i_kalinina1950@mail.ru \\ ${ }^{2}$ Научно-исследовательский отдел, АО Научно-Производственное Предприятие “Термотекс”, ул. Заводская, \\ д. 1. Московская обл. г. Хотьково, Российская Федерация, 141371 \\ E-mail: Alex_Bova@mail.ru
}

В статье приводятся результаты повышения прочности единичных филаментов сополимерного пара-арамидного волокна при обработке волокна многослойными углеродными нанотрубками. Эксперименты проводились на промышленной установке акционерного общества «Термотекс». Использовались многослойные углеродные нанотрубки производства Уральского научно-исследовательского института композиционных материалов. Окисление проводилось смесью концентрированных азотной и серной кислот. После отмывки кислот приготавливалась водная суспензия с использованием ультразвукового излучения. Водная суспензия окисленных многослойных нанотрубок обладала достаточной устойчивостью для проведения технологических экспериментов на серийном промышленном оборудовании. Определены параметры распределения Вейбулла для массивов экспериментальных данных прочности филаментов исходного волокна и филаментов образца волокна, обработанного многослойными углеродными нанотрубками. С использованием полученных результатов по методу Kelly и Туsоп рассчитаны предельные экстремальные значения прочности композитов, изготовленных из испытанных образцов волокон. Экстремальное значение для однонаправленного композиционного материала, изготовленного на основе исходного волокна, составило 5,699 ГПа, для композиционного материала на основе волокна, обработанного окисленными многослойными нанотрубками 6,270 ГПа. Увеличение составило 10,2 \%. Полученные результаты показывают возможность увеличения прочностных свойств, как волокна, так и композиционного материала на его основе без существенной модернизации собственно дорогостоящей химической технологии пара-арамидного волокна, что подчёркивает технико-экономическую перспективность направления.

Ключевые слова: пара-арамидное волокно, углеродные нанотрубки повышение экстремальной прочности композита

\section{INCREASING THE EXTREME VALUES OF THE STRENGTH OF ORGANOPLASTICS IN PROCESSING ARAMID FIBER WITH MULTILAYER CARBON NANOTUBES}

\author{
M.S. Shebanov ${ }^{1}$, V.B. Ivanov ${ }^{1}$, I.G. Kalinina ${ }^{1}$, V.G. Bova ${ }^{2}$, L.B. Shiyanova ${ }^{2}$ \\ ${ }^{1}$ Department of the dynamics of chemical and biological processes. N.N. Semenov Federal Research Center \\ for chemical physics Russian Academy of Science. 4 Kosygin Street, Moscow, Russian Federation, 119991 \\ E-mail: shebanov1989@gmail.com,vik93973@yandex.ru,i_kalinina1950@mail.ru \\ ${ }^{2}$ Research Department, JSC Scientific and Production Enterprise "Termoteks", st. Zavodskaya, 1, Khotkovo, \\ Moscow region, Russian Federation, 141371 \\ E-mail: Alex_Bova@mail.ru
}


The article presents the results of increasing the strength of single filaments of copolymer vapor of aramid fiber when processing the fiber with multilayer carbon nanotubes. The experiments were carried out on an industrial plant of the Termotex joint-stock company. We used multilayer carbon nanotubes of industrial production by the Ural Research Institute of Composite Materials. Oxidation was carried out with a mixture of concentrated nitric and sulfuric acids. After washing off the acids, an aqueous suspension was prepared using ultrasonic radiation. An aqueous suspension of oxidized multilayer nanotubes was sufficiently stable for carrying out technological experiments on commercial industrial equipment. The parameters of the Weibull distribution are determined for arrays of experimental data on the strength of filaments of the original fiber and filaments of a fiber sample treated with multilayer carbon nanotubes. Using the results obtained by the Kelly and Tyson method, the ultimate strength values of composites made from tested fiber samples are calculated. The extreme value for a unidirectional composite material based on the original fiber was $5.699 \mathrm{GPa}$, for a composite material based on a fiber treated with oxidized multilayer nanotubes it was $6.270 \mathrm{GPa}$. The increase was $10.2 \%$. The results obtained show the possibility of increasing the strength properties of both the fiber and the composite material made of it without significant modernization of the actual expensive chemical technology of aramid fiber vapor, which emphasizes the technical and economic prospects of the direction.

Key words: para-aramid fiber, carbon nanotubes, increase the extreme strength of the composite

Для цитирования:

Шебанов М.С., Иванов В.Б., Калинина И.Г., Бова В.Г., Шиянова Л.Б. Увеличение экстремальных значений предела прочности органопластиков при обработке арамидного волокна многослойными углеродными нанотрубками. Рос. хим. ж. (Ж. Рос. хим. об-ва). 2021. T. LXV. № 4. С. 3-7

For citation:

Shebanov M.S., Ivanov V.B., Kalinina I.G., Bova V.G., Shiyanova L.B. Increasing the extreme values of the strength of organoplastics in processing aramid fiber with multilayer carbon nanotubes. Ros. Khim. Zh. 2021. V. 65. N 4. P. 3-7

\section{ВВЕДЕНИЕ}

Полимерные волокна из поли- $n$-фенилентерефталамида (коммерческие названия волокон Kevlar (США), Twaron (Япония), Heracron (Корея), Taparan (Китай), Русар, Руслан (Россия)) обладают совокупностью свойств, которые позволяют использовать их в экстремальных условиях. На основе этих волокон выпускают, в частности, вертолётные лопасти и тканые (мягкие) бронежилеты для индивидуальной защиты, кордную нить для покрышек колёс гоночных автомобилей и самолётов. Отечественные арамидные волокна, выпускаемые АО "НПП "Термотекс" являются мировым лидером по прочностным характеристикам среди арамидных волокон, однако потенциал увеличения прочных свойств за счёт модернизации химической технологии почти полностью исчерпан и его дальнейшее развитие связано с необходимостью проведения длительных и дорогостоящих исследований. В настоящей работе приводятся результаты физико-химической модификации поверхности углеродного волокна многослойными углеродными нанотрубками (МУНТ) с целью повышения прочностных свойств волокна, а также прогноз увели- чения экстремальных значений прочности при растяжении однонаправленных органопластиков как результат увеличения прочности волокна при использовании МУНТ. Проблема увеличения прочностных свойств арамидных волокон и композитов из них (органопластиков) довольно остро стоит во всём мире. Изготовителями волокон ведутся интенсивные исследования, направленные прежде всего на модификацию поверхности готового волокна. В работе [1] теоретическими методами молекулярной динамики было показано, что при использовании в микроструктуре кевларового волокна функционализированных углеродных нанотрубок, прочность на разрыв его может быть увеличена на 27,8-39,7\%. В работе [2] модификации микрочастицами оксида кремния подвергалась уже готовая ткань из арамидного волокна Kevlar 49. Максимальный эффект упрочнения композиционного материала из модифицированной ткани наблюдали при содержании диоксида кремния $2 \%$. В работе [3] при обработке поверхности арамидного волокна Twaron окисленными многослойными нанотрубками было получено увеличение сдвиговых характеристик композита с полипропиленовой матрицей на 48,6\%. В работе [4] наряду с 
функционализацией углеродных нанотрубок модифицировалась химически и поверхность арамидного волокна. Использовались окисленные МУНТ, модификация поверхности арамидного волокна проводилась по методу алкилирования Фриделя Крафтса. Прочность собственно волокна не изменилась, но усилие выдергивания волокна из блока матрицы (метод pull-out) увеличилось на 53,11\%.

\section{ТЕОРЕТИЧЕСКАЯ ЧАСТЬ}

Оценка предельной прочности однонаправленного композиционного органопластика проводили по методу Kelly-Tyson [5, 6]. Обнаружено, что при разрушении КМ дробление единичных волокон идёт до определённого предела. Этот предел был назван критической длиной.

$$
L_{c r i t}=\frac{\sigma_{L} d_{f}}{2 \tau_{m}}
$$

Для волокон, используемых для создания КМ характерна зависимость прочностных свойств от базы измерений [7], причём с уменьшением базы измерений предел прочности растет, а значения модуля уменьшаются. При минимальном значении длины отрезка волокна, который может встретиться в КМ, его прочность, очевидно и будет максимальной. Уравнение (1), несмотря на свою простоту, часто используется в зарубежных исследованиях для анализа свойств КМ. В последнее два десятилетия появилось и интенсивно развивается новое направление исследований КМ, которое можно назвать экспериментальной микромеханикой [8], в котором уравнение (1) используется для определения числового значения напряжения на границе матрица-волокно. Уравнение (1) содержит три величины, которые заранее неизвестны, $\tau_{m}$ напряжение сдвига матрицы на границе с волокном и значение прочности $\sigma_{L}$, соответствующее значению $L_{\text {crit. }}$ В первом приближении можно принять для $\tau_{m}$ предел прочности при сдвиге эпоксидной смолы. Среднее значение можно принять исходя из результатов [10] 75 МПа. Средний диаметр единичного филамента волокна $d_{f}$ известен. В монографии [11] подчёркивается, что прочность полимерных волокон имеет статистическую природу и для оценки средней прочности волокна при произвольной длине базы измерений $L$ предлагается использовать распределение Вейбулла. Функция распределения Вейбулла F имеет вид:

$$
F(\sigma)=1-e^{-\frac{L}{L_{0}}\left(\frac{\sigma}{m_{a}}\right)^{\alpha}}
$$

где $L_{0}$ - некоторое значение базы измерений прочности, при котором проведены измерения для определения параметров распределения, $m_{a}$ (параметр масштаба) и $\alpha$ (параметр формы). Для расчета среднего значения прочности при произвольной длине $L$ получена [11] формула:

$$
\sigma(L)=m_{a}\left(\frac{L_{0}}{L}\right)^{1 / \alpha} \Gamma(1+1 / \alpha)
$$

где $\Gamma$ - гамма функция Эйлера. Подставив функцию (3) в уравнение (1) можно определить значение критической длины и затем экстремальное (предельное) значение однонаправленного органопластика.

\section{ЭКСПЕРИМЕНТАЛЬНАЯ ЧАСТЬ}

Образцы сополимерного арамидного волокна были получены в производственных условиях в АО "НПП “Термотекс". Для модификации волокна использовались многослойные углеродные нанотрубки (МУНТ) производства Уральского НИИ композиционных материалов (г. Пермь). Окисление МУНТ производилось смесью концентрированных азотной и серных кислот, взятых в соотношении 1:3 при перемешивании стеклянной мешалкой в течение 3 ч. Для активации суспензия подвергалась воздействию ультразвука. Использовался титановый излучатель, мощность генератора составляла 800 Вт. Аналогичные методики использовались в работах $[3,4,9]$. Измерения прочности филаментов проводились на разрывной машине Textechno (Германия). Измерения проводились на базе 10 мм (так называемая зажимная длина).

\section{РЕЗУЛЬТАТЫ И ИХ ОБСУЖДЕНИЕ}

Для обработки экспериментальных данных использовался универсальный математический пакет Matlab. Результаты первичной статистической обработки экспериментальных данных приведены в табл. 1. Под средними значениями указаны нижняя и верхняя границы доверительных интервалов. Нижние и верхние границы доверительных интервалов рассчитывались с использованием критерия Стьюдента соответственно:

$$
x_{c p}-\frac{t(\alpha)}{\sqrt{N}} s \ldots \ldots x_{c p}+\frac{t(\alpha)}{\sqrt{N}} s
$$

где $x_{c p}$-среднее значение массива экспериментальных данных, $t(\alpha)$ - значение критерия Стьюдента при уровне значимости $\alpha$, который принимался равным $0,05, N$ - число параллельных экспериментов.

Во всех случаях доверительные интервалы для свойств филаментов исходного волокна не пересекаются с доверительными интервалами для свойств филаментов волокна, обработанного МУНТ. Это означает, что разница в свойствах статистически значима, а реальные волокна представляют собой фактически различные материалы. Значения параметров распределения Вейбулла и их 
доверительные интервалы, рассчитывались с помощью стандартной процедуры пакета Matlab, однако требуется проверка возможности применения данного распределения к полученным экспериментальным данным. Для этой цели используем непараметрический критерий согласия Колмогорова теоретической и выборочной (экспериментальной, эмпирической) функции распределения. Выборочная (эмпирическая) функция распределения $F_{n}\left(x_{i}\right)$ рассчитывается только из экспериментальных данных:

$$
F_{n}\left(x_{i}\right)=\left\{\begin{array}{l}
0 \quad x \leq x_{1} \\
\cdots \cdots \cdots \\
i / N \quad x_{i} \leq x \leq x_{i+1} \\
\cdots \cdots \\
(N-1) / N \quad x_{N-1} \leq x \leq x_{N} \\
1 \quad x \geq x_{N}
\end{array}\right.
$$

\section{Значения прочностных свойств единичных} филаментов

Под средними значениями указаны нижняя и верхняя границы доверительных интервалов

\begin{tabular}{|c|c|c|c|c|c|}
\hline \multirow{2}{*}{ Образец } & \multicolumn{2}{|c|}{ прочность, ГПа } & \multicolumn{2}{|c|}{ модуль, ГПа } & \multirow{2}{*}{$\mathrm{N}$} \\
\hline & \multicolumn{2}{|c|}{ среднее } & \multicolumn{2}{|c|}{ среднее } & \\
\hline \multirow{2}{*}{$\begin{array}{c}\text { исход- } \\
\text { ный }\end{array}$} & \multicolumn{2}{|c|}{4,623} & \multicolumn{2}{|c|}{94,01} & \multirow{2}{*}{29} \\
\hline & 4,383 & 4,862 & 91,97 & 96,04 & \\
\hline Обрабо- & \multicolumn{2}{|c|}{5,241} & \multicolumn{2}{|c|}{98,86} & \multirow[b]{2}{*}{24} \\
\hline $\begin{array}{l}\text { танный } \\
\text { МУНТ }\end{array}$ & 5,034 & 5,4483 & 96,88 & 100,83 & \\
\hline
\end{tabular}

Критерии согласия накладывают определённые условия на "расстояние" между эмпирической и теоретической функциями распределения. Выполнение или невыполнение этих условий позволяет либо принять (согласие) либо отвергнуть проверяемое распределение. В качестве "расстояния" критерий Колмогорова использует модуль разности между теоретической и эмпирической функциями распределения [12]:

$$
D_{n}=\sup \left|F_{n}(x)-F(x)\right|
$$

Для конкретного значения $D_{n}$ может быть определена вероятность:

$$
P\left[\frac{\left(6 N D_{n}+1\right)^{2}}{18 N}<x\right]=K\left(\sqrt{\frac{x}{2}}\right)
$$

где К-распределение Колмогорова:

$$
K(y)=\sum_{k=-\infty}^{\infty}(-1)^{k} e^{-k^{2} y^{2}}
$$

Для практического использования рекомендуются [12] формулы:

$$
\begin{aligned}
& D_{n}^{+}=\max \left(\frac{i}{N}-F\left(x_{i}\right)\right) \\
& D_{n}^{-}=\max \left(F\left(x_{i}\right)-\frac{i-1}{N}\right) \\
& D_{n}=\max \left(D_{n}^{+}, D_{n}^{-}\right)
\end{aligned}
$$

Если значение $D_{n}$ определенное из экспериментальных данных по формулам (9а-9в), окажется больше критического значения $D_{n}(\alpha)$, определённого для заданного уровня значимости $\alpha$, согласно критерию Колмогорова с уровнем значимости $\alpha$ гипотеза согласия экспериментальных данных и теоретического распределения отвергается. Для определения критического значения $D_{n}(\alpha)$ для практических целей получена аппроксимация:

$$
D_{n}(\alpha)=\sqrt{\frac{-\ln (0.5 \alpha)}{2 N}}-\frac{1}{6 N}
$$

где $\alpha$-заданный (обычно 0,05 ) уровень значимости. Для филаментов исходного волокна экспериментальное значение $D_{n}=0,1649$, для обработанных МУНТ $D_{n}=0,1856$. Критическое значение в обоих случаях составило $D_{n}(\alpha)=0,3753$. Гипотеза согласия экспериментальных данных и теоретического распределения Вейбулла не отвергается. Значения параметров распределения и их доверительные интервалы, определённые с использованием стандартной программы wblfit универсального пакета Matlab приводятся в табл. 2.

Таблица 2

Параметры распределения Вейбулла

\begin{tabular}{|c|c|c|c|c|}
\hline \multirow{2}{*}{ Образец } & \multicolumn{3}{|c|}{ Параметры распределения Вей- } \\
\cline { 2 - 4 } & \multicolumn{3}{|c|}{ булла } \\
\cline { 2 - 4 } & \multicolumn{2}{|c|}{ среднее } & \multicolumn{2}{|c|}{ фрермане } \\
\hline \multirow{2}{*}{ Исходный } & \multicolumn{2}{|c|}{4,8676} & \multicolumn{2}{|c|}{10,1998} \\
\cline { 2 - 5 } & 4,6748 & 5,0682 & 7,3839 & 14,0897 \\
\hline $\begin{array}{c}\text { Обработан- } \\
\text { ный } \\
\text { МУНТ }\end{array}$ & \multicolumn{2}{|c|}{5,4665} & \multicolumn{2}{|c|}{11,5358} \\
\cline { 2 - 5 } & 5,2690 & 5,6714 & 8,5133 & 15,6313 \\
\hline
\end{tabular}

Как следует из результатов, представленных в табл. 1, увеличение средней прочности филаментов после обработки волокон МУНТ для критической зажимной длины составило 13,38\%. Значения критической длины для исходного и обработанного МУНТ филаментов составило 1,215 и 1,236 мм соответственно. Этим значениям соответствуют экстремальные значения предела прочности однонаправленного композита 5,699 ГПа и 6,270 ГПа соответственно. Увеличение экстремального значения прочности композита составило $10,2 \%$. 


\section{ВЫВОДЫ}

В результате использования при обработке арамидного волокна окисленных МУНТ получено увеличение прочности единичных филаментов на $13,38 \%$. (Аналогичный результат в доступной научно-технической литературе не обнаружен).

Соответствующие экстремальные значения предела прочности, рассчитанные по методу KellyTyson составили для исходного волокна 5,699 ГПа и волокна, обработанного окисленными МУНТ 6,270 ГПа соответственно, увеличение составило $10,2 \%$.

Полученные эффекты показывают возможность увеличения прочностных свойств как волокна, так и композиционного материала на его основе без существенной модернизации собственно дорогостоящей химической технологии волокна, что подчёркивает технико-экономическую перспективность направления.

Работа выполнена в соответствии с Госзаданием № 0082-18-006, номер государственной регистрации АААA-A18-118020890097-1.

\section{ЛИТЕРАТ УР А REFERENCES}

1. Zebei Mao, Tong Li, Ke Zhang, Dongyu Li, Caihua Zhou, Mingfa Ren, Yuantong Gu, and Bo Wang. "Carbon Nanotube Reinforced Poly- p- Phenylene Terephthalamide Fibers for Toughness Improvement: A Molecular Dynamics Study." Advanced Theory and Simulations. 2020. V. 3.10. P. 2000135. DOI.org/10.1002/adts.202000135.

2. Sahab Uddin, Shariful Islam, Farjana Showline Chaity, Ali Akbar, Shahin Akand, Gafur M. A., Sarwaruddin A.M. Evaluation of Laminated Composites Reinforced by High-performance Kevlar Filaments with Variable SiO2: Mechanical, Morphological \& Thermal Tests. Composite Materials. 2020. V. 4(2). P. 15-24. DOI: 10.11648/j.cm.20200402.11.

3. Gonzalez P.I., Rodríguez-Uicab O., Martin-Barrera C., UribeCalderon J., Canché-Escamilla G., Yazdani-Pedram M., May-
Pata A., Avilésa F. Influence of aramid fiber treatment and carbon nanotubes on the interfacial strength of polypropylene hierarchical composites. Composites Part B: Engineering. 1 August 2017. V. 122. P. 16-22. DOI.org/10.1016/j.compositesb.2017.04.006.

4. Yang X., Tu Q., Shen X., Zhu P., Li Y., Zhang S. A novel method for deposition of multi-walled carbon nanotubes onto poly (p-phenylene terephthalamide) fibers to enhance interfacial adhesion with rubber matrix. Polymers. 2019. V. 11(2). P. 374. DOI: 10.3390/polym1 1020374.

5. Aliotta L., Lazzeri A. A proposal to modify the Kelly-Tyson equation to calculate the interfacial shear strength (IFSS) of composites with low aspect ratio fibers. Composites Science and Technology. 20 January 2020. V. 186. P. 107920. DOI: 10.1016/j.compscitech.2019.107920.

6. Zhan-Ying Sun, Hai-Shan Han. Gan-Ce Dai. Mechanical Properties of Injection-molded Natural Fiber-reinforced Polypropylene Composites: Formulation and Compounding Processes. Journal of Reinforced Plastics and Composites. 2010. V. 29(5). P. 637-650 DOI: 10.1177/0731684408100264.

7. Zhu D., Mobasher B., Erni J., Bansal S., Rajan S.D. Strain rate and gage length effects on tensile behavior of Kevlar 49 single yarn. Composites Part A: Applied Science and Manufacturing.V. 43, Issue 11, November. 2012. P. 2021-2029. DOI: 10.1016/j.compositesa.2012.06.007.

8. Liu X.Y., Dai G.C. Surface modification and micromechanical properties of jute fiber mat reinforced polypropylene composites. eXPRESS Polymer Letters. 2007. V. 1. No.5. P. 299-307. DOI: 10.3144/expresspolymlett.2007.43.

9. Ramanathan T., Fisher F.T., Ruoff R.S., Brinson L.C. Amino-Functionalized Carbon Nanotubes for Binding to Polymers and Biological Systems. Chem. Mater. 2005. V. 17. N. 6. P. 1290-1295. DOI: 10.1021/cm048357f.

10. Rich M.J., Drzal L.T., Hunston D., Holmes G., McDonough $W$. Round robin assessment of the single fiber fragmentation test. In Procedings of the American Society for Composites 17th Technical Conference. CRC Press. 2002. P. 158-182.

11. Работнов Ю.Н. Механика деформируемого твердого тела. М.: Наука. 1988. 712 с. Rabotnov Y.N. Mechanics of a Deformable Solid Body. Moscow.: Nauka. 1988. 712 p.

12. Орлов А.И. Непараметрические критерии согласия Колмогорова, Смирнова, омега-квадрат и ошибки при их применении. Научный журнал КубГАУ. 2014. № 97(03). С. 644-672. Orlov A.I. Nonparametric goodness-of-fit Kolmogorov, Smirnov, omegasquadrate test and errors in their application. Scientific Journal of KubSAU. 2014. № 97(03). P. 644-672.

Поступила в редакиию (Received) 01.08.2021

Принята к опубликованию (Accepted) 03.09.2021 